\title{
A systematic approach to the design of buffer tanks
}

\author{
Audun Faanes *,1, Sigurd Skogestad \\ Department of Chemical Engineering, Norwegian University of Science and Technology, N-7491 Trondheim, Norway
}

\begin{abstract}
Buffer tanks are often designed and implemented for control purposes, yet control theory is rarely used when sizing and designing buffer tanks and their control system. Instead, rules of thumb such as using ' 10 min residence time' are used. The objective of this paper is to provide a systematic approach. We consider mainly the case where the objective of the buffer tank is to dampen ('average out') the fast (i.e. high frequency) disturbances, e.g. in flow and concentration, which cannot be handled by the feedback control system. (C) 2000 Elsevier Science Ltd. All rights reserved.
\end{abstract}

Keywords: Process control; Processs design; Buffer tanks

\section{Introduction}

The objective of this paper is to provide a systematic approach to the design of buffer tanks based on control theory. The background for this approach is that buffer tanks are often implemented for control purposes. Even so, control theory is rarely used when sizing and designing the tanks. Instead, rules of thumb are used.

Text books on chemical process design seems to agree that a half-full residence time of $5-10 \mathrm{~min}$ is appropriate for reflux drums and that this also applies for other buffer tanks. For tanks between distillation columns a half-full residence time of $10-20 \mathrm{~min}$ is recommended (Lieberman, 1983; Ulrich, 1984; Wells, 1986; Sandler \& Luckiewicz, 1987; Walas, 1987). Sigales (1975) is more specific concerning what follows after the drum. None of these references give any justifications for their choice. Watkins (1967) gives a reflux drum volume dependent on instrumentation and labor factors (both related to operational use of the buffer tank), reflux and product rates, and a factor dependent on how well external units are operated. The method gives half full hold-up times from 1.5 to 32 min.

Design of vessels to dampen flow variations is presented by Harriott (1964) using a specification of outlet

\footnotetext{
* Corresponding author.

E-mail address: skoge@chembio.ntnu.no (S. Skogestad).

${ }^{1}$ Also affiliated with Norsk Hydro ASA.
}

flow rate change given a certain step in inlet flow. This method has similarities with the one presented for flow variations in the present paper.

Another related class of process equipment is neutralization tanks. The main problems for this process are large and varying process gain and delays in the control loop. Design is described in Shinskey (1973) and McMillan (1984). Another design method and a critical review is found in Walsh (1993).

Zheng and Mahajanam (1998) find the necessary buffer tank volume by optimization and use it as a controllability measure.

A stated above, due to limitations in the control system, there is a limitation in frequencies above which the control system is not effective. The process itself must dampen the disturbances in this area. If it initially does not, addition of one or more buffer tanks is necessary. In this paper we present design methods for buffer tanks based on this fundamental understanding.

\section{Transfer functions for buffer tanks}

Consider the effect of a disturbance, $d$, on the control variable $y$. The linearized model in terms of deviation variables may be written as

$y(s)=G_{\mathrm{d}}(s) d(s)$

To illustrate the effect of the buffer tank, we express the dynamic model of the tank with the transfer functionfl 
$h(s)$. The disturbances passes through the buffer tank (e.g. see Fig. 1), so that the process with a buffer tank may be expressed by

$$
G_{\mathrm{d}}(s)=G_{\mathrm{d}_{0}}(s) h(s)
$$

where $G_{\mathrm{d}_{0}}(s)$ is the disturbance transfer function of the original plant, and $G_{\mathrm{d}}(s)$ is the modified disturbance transfer function. A typical buffer tank transfer function is

$h(s)=1 /(\tau s+1)$

Note that $h(0)=1$ so that the buffer tank has no steady state effect.

We consider a buffer tank with liquid volume $V\left(\mathrm{~m}^{3}\right)$, in-let flow-rate $q_{\text {in }}\left(\mathrm{m}^{3} / \mathrm{s}\right)$, outlet flow-rate $q$. Further, we let $c_{\text {in }}$ and $c$ denote the inlet and outlet quality (concentration or temperature), respectively. A component or simplified energy balance for a perfectly mixed tank yields

$\mathrm{d}(V c) / \mathrm{d} t=q_{\mathrm{in}} c_{\mathrm{in}}-q c$

In addition we have the total mass balance (assuming constant density):

$\mathrm{d} V / \mathrm{d} t=q_{\text {in }}-q$

\subsection{Quality disturbance}

For quality disturbances the objective of the buffer tank is to smoothen the quality response, $c(s)=$ $h(s) c_{\text {in }}(s)$, so that the variations in $c$ are smaller than in $c_{\text {in }}$. Combining (4) and (5) yields $V(\mathrm{~d} c / \mathrm{d} t)=q_{\text {in }}\left(c_{\mathrm{in}}-c\right)$ and for a single buffer tank linearization yields

$c(s)=\frac{1}{\frac{V *}{q *} s+1}\left[c_{\mathrm{in}}(s)+\frac{c *_{\mathrm{in}}-c *}{q *} q_{\mathrm{in}}(s)\right]$

where ${ }^{*}$ denotes the nominal (steady state) values. Note that the dynamics of $V$ (level control) have no effect on the linearized response of $c$. Furthermore, for the case with a single feed stream $c_{\mathrm{in}}^{*}=c^{*}$, the dynamics of $q_{\mathrm{in}}$ have no effect on the response and the dynamics of $c$. In any case we find that the transfer function for quality is

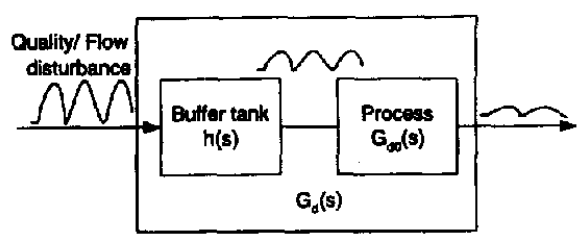

Fig. 1. Example of how a buffer tank dampens disturbances. $h(s)=1 /\left(\tau_{\mathrm{h}} s+1\right)$

where $\tau_{\mathrm{h}}=V^{*} / q^{*}(\mathrm{~s})$ is called the residence time (steady state). We can see that the buffer tank works as a first order filter. Similarly for $n$ buffer tanks in series we have

$h(s)=1 /\left(\frac{\tau_{h}}{n} s+1\right)^{n}$

where $\tau_{\mathrm{h}}$ is the total residence time.

\subsection{Flow rate disturbance}

For flow rate disturbances the objective of the buffer tank is to smoothen the flow response, $q(s)=h(s) q_{i n}(s)$. Note that we need to use a 'slow' level controller, as tight level control yields $q \approx q_{\text {in }}$. Let $k(s)$ denote the transfer function for the level controller including measurement and actuator dynamics and the possible dynamics of an inner flow control loop. Then $q(s)=k(s)\left(V(s)-V_{\mathrm{s}}\right)$, where $V_{\mathrm{s}}$ is the set-point for the volume. Combining this with the total mass balance (5) yields

$q=\frac{k(s)}{s+k(s)} q_{\mathrm{in}}(s)-\frac{s k(s)}{s+k(s)} V_{\mathrm{s}}$

The buffer tank transfer function is thus given by

$h(s)=\frac{k(s)}{s+k(s)}=\frac{1}{\frac{s}{k(s)}+1}$

In this case we have more freedom in selecting $h(s)$ since we can select the controller $k(s)$. With a proportional controller $k(s)=K$, we get that $h(s)$ is a first order filter with $\tau=1 / K$. For a given $h(s)$ the controller is

$k(s)=\operatorname{sh}(s) /(1-h(s))$

\section{Controllability analysis}

We here provide a review of some controllability results which are subsequently used for buffer tank design. We consider SISO (single input-single output) systems. Consider a linear process in terms of deviation variables

$y(s)=G(s) u(s)+G_{\mathrm{d}}(s) d(s)$

Here $y$ denotes the output, $u$ the manipulated input and $d$ the disturbance (including disturbances entering at the input, which are frequently referred to as load changes'). We assume throughout this paper that the model has been scaled such that expected disturbances make the magnitude of the elements of $d$ lie within \pm 1 for all frequencies and the requirement for the scaled output vector, $y$, is that the magnitude of each element in $y$ shall lie between -1 and 1 for all frequencies, and 
$u$ is scaled so that the manipulated input range corresponds to a variation of \pm 1 in $u$.

Feedback control yields $u(s)=K(s)\left(y_{s}(s)-y(s)\right)$, and from this we eliminate $u$ to get

$$
\begin{aligned}
y(s) & =\frac{G(s) K(s)}{1+G(s) K(s)} y_{\mathrm{s}}(s)+\frac{G_{\mathrm{d}}(s)}{1+G(s) K(s)} d(s) \\
& =T(s) r(s)+S(s) G_{\mathrm{d}}(s) d(s)
\end{aligned}
$$

$y_{\mathrm{s}}$ is the set-point, and $S(s)$ and $T(s)$ are the sensitivity function and the complementary sensitivity function, respectively. We ignore set-point changes and get the following expression for the effect of disturbances

$y(s)=S(s) G_{\mathrm{d}}(s) d(s)$

Two different requirements must be fulfilled to get acceptable control performance. The first relates to the speed of response to reject disturbances. From (14) we see that to keep $|y|<1$ when $|d|=1$, we must require

$\left|S(j \omega) G_{\mathrm{d}}(j \omega)\right| \leq 1 ; \quad \forall \omega$

We define $\omega_{\mathrm{B}}$ as the frequency where $|S(j \omega)|=1$. At higher frequencies we cannot rely on feedback control for disturbance rejection, so that

$\left|G_{\mathrm{d}}(j \omega)\right| \leq 1 ; \quad \omega \geq \omega_{\mathrm{B}}$

For acceptable performance and robustness we have the following maximum value of the bandwidth (Skogestad \& Postlethwaite, 1996; Skogestad, 1999):

$\omega_{\mathrm{B}}=1 / \theta_{\text {cff }}$

where $\theta_{\text {efr }}$ is the effective delay. With PI or PID control we have (Skogestad, 1999):

$\theta_{\text {eff }}=\theta+\tau_{z}+\frac{\tau_{j}}{2}+\sum_{i>j} \tau_{i} ; \quad j=2$ for PI

where $\theta$ is the delay, $\tau_{z}=1 / z$, where $z$ is a right halfplane zero, and $\tau_{i}$ is lag number $i$ ordered by size so that $\tau_{1}$ is the largest time constant. For more realistic PI controllers (17) may be modified. Ziegler-Nichols tuning gives $\omega_{\mathrm{B}}=1 /\left(1.31 \theta_{\mathrm{erf}}\right)$, while a more robust tuning (Skogestad, 1999) gives

$\omega_{\mathrm{B}}=1 /\left(20_{\mathrm{eff}}\right)$

Note that (16) is only a necessary requirement, as (15) needs to be satisfied for $\omega<\omega_{\mathrm{B}}$. In particular, (15) may impose additional requirements if $G_{\mathrm{d}}$ is of high order; this is discussed later.

In words (16) tells us that at sufficiently high frequencies the process must be 'self-regulating'. If (16) is not satisfied then we need to modify the process. One commonly used approach is to add buffer tanks as illustrated in Fig. 1, such that the 'new' disturbance response becomes as in Eq. (2).

The second limitation relates to input constraints for disturbances, but will not be covered by this article.

\subsection{Additional requirements due to high order}

As mentioned, (16) is only a necessary requirement as (15) also needs to be satisfied for $\omega<\omega_{\mathrm{B}}$. To investigate this further we made the following approximation of the sensitivity function, $S(j \omega)$, with the loop transfer function, $L(j \omega)(=G(j \omega) K(j \omega))$ :

$S(j \omega)=1 /(1+L(j \omega)) \approx 1 / L(j \omega)$

Inserting this approximation into (15), we obtain

$\left|G_{\mathrm{d}}(j \omega)\right| \leq|L(j \omega)| ; \quad \forall \omega$

Now it may be difficult to have sufficiently high roll-off (slope) in the loop transfer function $L(s)$ to get $|L(j \omega)| \geq\left|G_{\mathrm{d}}(j \omega)\right|$ at frequencies below the bandwidth (even though we satisfy it at the bandwidth). The problem is that a high roll-off in $L(s)$ yields a large phase lag, and we get instability problems. For reasonable robustness and performance we must have that the slope for $|\mathrm{L}|$ at about -1 near the bandwidth $\omega_{\mathrm{B}}$. In this case it is difficult to make general formulas for the buffer tank design. Graphical or optimization based solutions are probably simplest. One particular case is studied later.

We can get a steeper slope around the bandwidth, however, with multiple control loops. For example with a series of $n$ buffer tanks and control in each tank, the total slope of $|L|$ is $-n$ (even though it is -1 for each individual tank).

\section{Quality variations}

When the main source of disturbances are variations in the inflow quality (temperature or concentration) they may be smoothened by a mixing tank. With perfect mixing and a residence time of $\tau_{\mathrm{h}}$ (denotes hold-up), the outflow quality is roughly speaking the sliding mean of the input quality within a time window of length $\tau_{\mathrm{h}}$. The transfer function for one buffer tank is given by (7). We may also consider using a series of buffer tanks. For $n$ equal tanks in series with a total residence time of $\tau_{\mathrm{h}}$, and total volume $V$, the transfer function is given by (8).

In Fig. 2 we show the amplitude plot of $h(s)$ for $n=1,2,3,4$ equal tanks in series with a given total residence time $\tau_{\mathrm{h}}$. Physically, on the $x$-axis is shown the normalized frequency, $\omega \tau$, of the sinusoidal varying input concentration,

$c_{\mathrm{in}}(t)=c_{\mathrm{in}, 0}(t) \sin (\omega t)$

into the first tank, and on the $y$-axis is shown the normalized output concentration from tank $n, c / c_{\text {in }, 0}$, where $c_{\mathrm{in}, 0}$ and $c_{0}$ denote the magnitude of the sinusoidal variations. Note that both axis are logarithmic. 


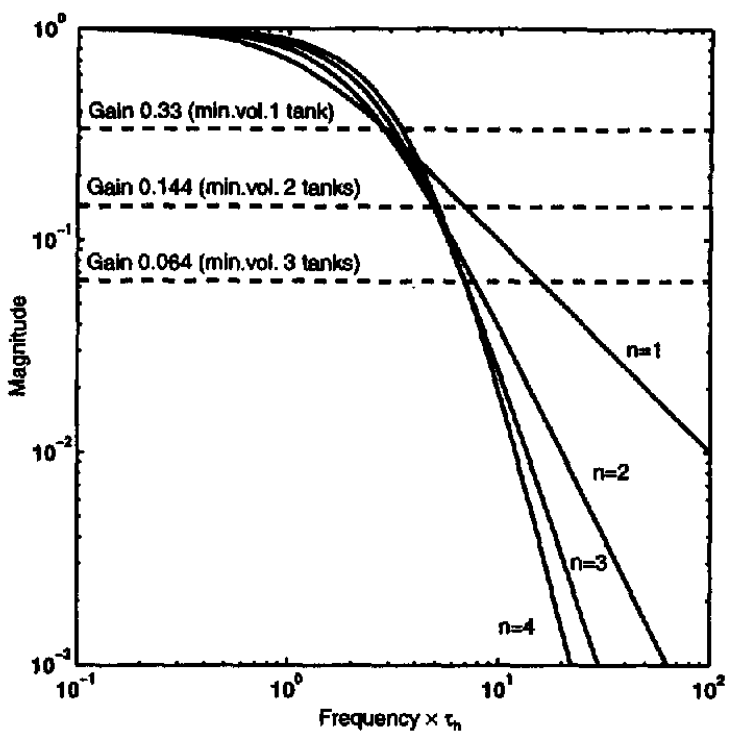

Fig. 2. Frequency responses for $n$ tanks in series with total residence time $\tau_{\mathrm{h}}, h(s)=1 /\left(\frac{\tau_{h}}{n} s+1\right)^{n}$.

At low frequencies, $\omega \ll 1 / \tau$, we have $c_{0} / c_{\text {in }} \approx 1$, which means that slow sinusoidal variations are unaffected when they pass through the tanks. However, fast variations (with high frequencies) are dampened by the tanks which tend to 'average out' the variations. At sufficiently high frequencies, $\omega \gg 1 / \tau$, we find that $c_{0} / c_{\mathrm{in}, 0}$ (log-scale) as a function of frequency (log-scale) approaches a straight line. This follows because the highfrequency asymptote is $|h(j \omega)|=\tau^{-n} \cdot \omega^{-n}$ (in words, 'the slope is $-n$ ' at high frequencies for $n$ tanks in series). Thus, at high frequencies the use of many tanks is 'better', in terms of providing more dampening for a given total volume.

On the other hand, the frequency where the asymptote crosses magnitude 1 (its 'break' or 'corner' frequency) is $\omega=1 / \tau=n / \tau_{\mathrm{h}}$, which is at a lower frequency when $n$ is smaller, so at lower frequencies fewer tanks is better. This is also seen from the more exact plot in Fig. 2.

The plot may be used to obtain the total required volume of the buffer tanks if we at a given frequency specify the $f$ factor by which we want to reduce the disturbance. The required 'gain' of the buffer transfer function is then $1 / f$ and we can read off $\omega \tau_{\mathrm{h}}$ and with a given value of $\omega$ obtain the total residence time $\tau_{\mathrm{h}}$. Typically, the given frequency is the achievable closedloop bandwidth of the feedback control system, $\omega=1 /$ $0_{\mathrm{eff}}$, and $f$ is the value of $G_{\mathrm{d}_{\mathrm{o}}}$ at this frequency.

We see that one tank is 'best' if we want to reduce the effect of the disturbance at a given frequency by a factor $f=3=1 / 0.33$ or less; two tanks are 'best' if the factor is between 3 and about $7=1 / 0.144$, and three tanks are 'best' if the factor is between about 7 and $15=1 / 0.064$.
The word 'best' has been put in quotes because we here only consider the total combined volume of the tanks. In practice, there are several other factors that favor using as few tanks as possible; this includes the scaling law for cost (typically, cost scales with $V^{0.7}$ ), the cost of additional equipment like pipes, pumps, sensors, control systems, etc. as well as other controllability considerations (slope condition on $L$ ). Therefore, one would also probably consider using only one tank when we want to reduce the effect of the disturbance by a factor $f=100$, even though in this case the volume of one tank is about five times larger than the total volume of two tanks, and more than seven times larger than the total volume of three tanks (this is seen from Fig. 2 by reading off the value of $\omega \tau_{h}$ that corresponds to magnitude $10^{-2}$ ).

To satisfy the necessary condition (16) we need to select $h(s)$ such that

$\left|h\left(j \omega_{\mathrm{B}}\right)\right|\left|G_{\mathrm{d} 0}\left(j \omega_{\mathrm{B}}\right)\right| \leq 1$

We introduce the factor by which the effect of the disturbance must be reduced

$f=\left|G_{\mathrm{d} 0}\left(j \omega_{\mathrm{B}}\right)\right|$

We must at least require $\left|h\left(j \omega_{\mathrm{B}}\right)\right|=1 /$ f. As mentioned this may be solved graphically using Fig. 2, but alternatively we can find the analytical solution from (8) and (17):

$\tau_{\mathrm{h}}>\theta_{\text {eff }} n \sqrt{f^{2 / n}-1}$

For one tank and $f \gg 1$ we have the appropriate formula $\tau_{\mathrm{h}}>f \theta_{\mathrm{efr}}$. For $n \geq 2$ the use of (24) assumes that the total slope of $|L|$ around $\omega_{\mathrm{B}}$ can be $-n$. This can be achieved with local quality control in each tank, e.g. for a neutralization plant, it must be possible to measure the concentration and automatically add a reactant in each tank.

To find the optimal number of tanks one must then take into account equipment, piping, control systems (each tanks may require a level controller) etc. as mentioned above. Normally the optimal number of tanks will not be large, so that the cost calculations has to be made for a limited number of cases.

Example 1. Consider the mixing of two process streams, A and B as illustrated in Fig. 3. The concentration and flow rate of stream $\mathrm{A}$ are denoted by $c_{\mathrm{A}}$ and $q_{\mathrm{A}}$, and for $\mathrm{B}$ they are called $c_{\mathrm{B}}$ and $q_{\mathrm{B}}\left(c_{\mathrm{A}}\right.$ and $c_{\mathrm{B}}$ may also be temperatures). The two streams are mixed in a mixing tank of $1 \mathrm{~m}^{3}$, and the concentration of the outlet flow is denoted $c_{0}$. This concentration $c_{0}$ is controlled by manipulating the flow rate of $B$. First we check if this controller, together with the mixing tank, is sufficient for suppressing disturbances in the concentration of stream A. Combination of component balance and total material balance gives the following model: 
$\frac{\mathrm{d} c_{0}}{\mathrm{~d} t}=\frac{1}{V}\left[\left(c_{\mathrm{A}}-c\right) q_{A}+\left(c_{B}-c\right) q_{B}\right]$

This model is linearized and scaled (as described in the controllability section). We require a variation in $c$ less than $1 / 10$ of the variation in $c_{\mathrm{A}}$, and the flow range of $q_{\mathrm{B}}$ is twice the flow range of $q_{\mathrm{B}}$. The scaled deviation variables are marked with a prime and we get the following model after Laplace transformation

$c^{\prime}(s)=\frac{1}{1+s}\left[10 c_{A}^{\prime}(s)+10 q_{A}^{\prime}(s)-20 q_{B}^{\prime}(s)\right]$

where we have assumed constant $c_{B}$. We study concentration disturbances leading to $G_{\mathrm{d}_{0}}(s)=10 /(1+s)$ and further $G_{0}(s)=-20 /(1+s)$. Mainly due to the mea-

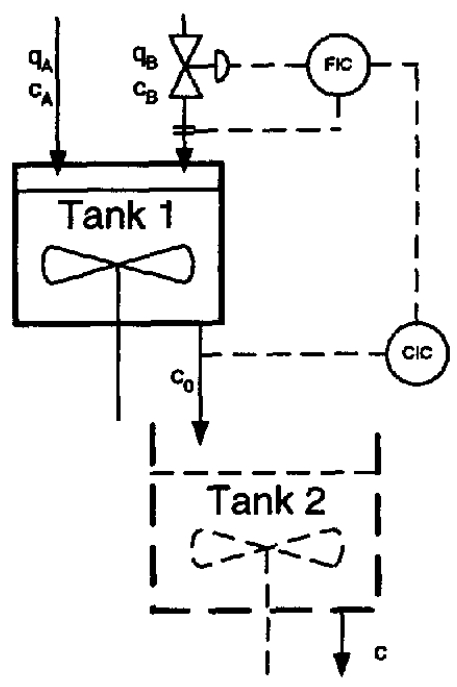

Fig. 3. Extra buffer tank for a mixing process. Concentration is controlled by manipulating flow rate of stream $\mathbf{B}$.

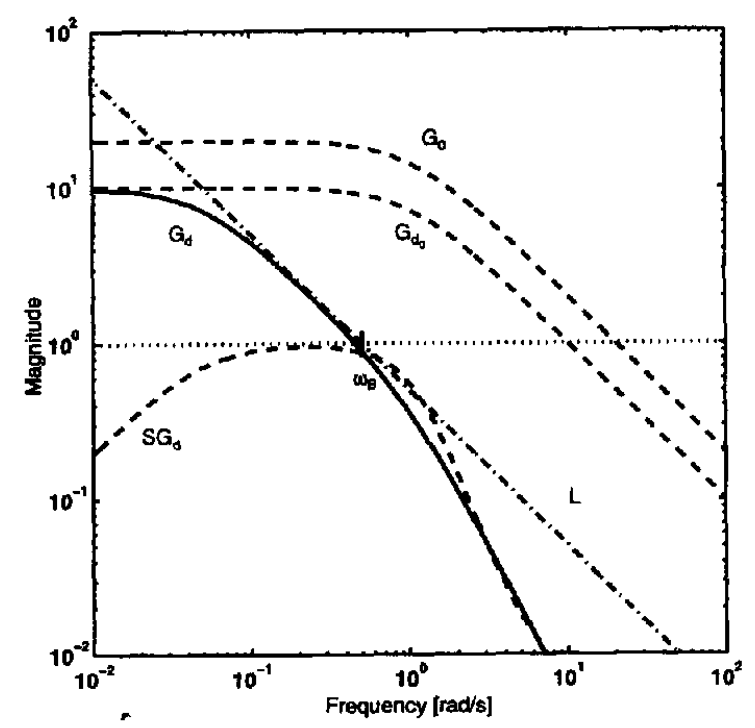

Fig. 4. With an extra buffer tank, $\left|G_{\mathrm{d}}\right|$ is brought below 1 for all frequencies above the bandwidth.

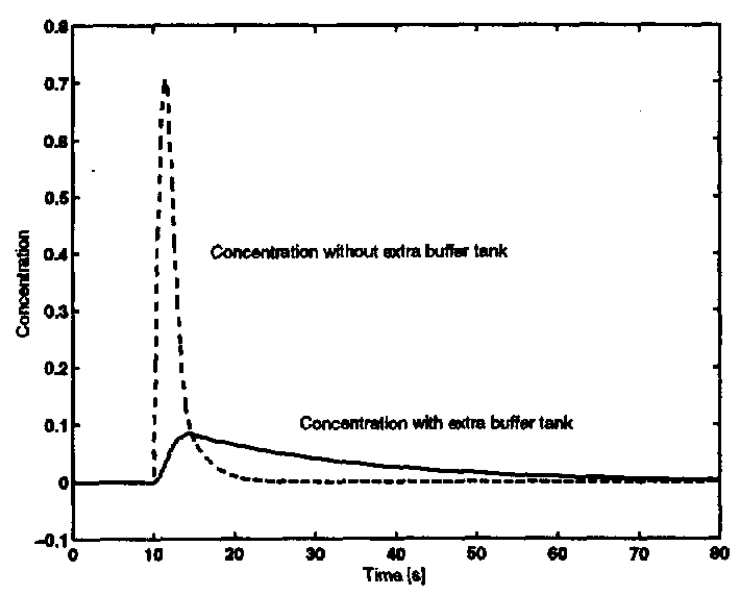

Fig. 5. With an extra buffer tank the outlet concentration is kept within 0.1 from set-point despite a unit step in disturbance. This is not the case without the extra buffer tank.

surement, the control loop has an effective delay of $1 \mathrm{~s}$. With a robust controller tuning, (19) gives a bandwidth of $0.5 \mathrm{red} / \mathrm{s}$.

$\left|G_{0}(j \omega)\right|$ and $\left|G_{\mathrm{d}_{0}}(j \omega)\right|$ are shown in Fig. 4 (dashed lines). We see that $\left|G_{0}\right|>\left|G_{\mathrm{d}_{0}}\right|$ for all frequencies, so that input constraints pose no problems in this case. In the figure the bandwidth frequency, $\omega_{B}$, is also marked.

We see that $\left|G_{\mathrm{d}_{0}}\right|>1$ even for frequencies above the bandwidth, so the control system is not sufficient to fulfil the requirements on the outlet concentration. To solve this problem, we may either improve the control system, increase the volume of the mixing tank from 1 $\mathrm{m}^{3}$ or install an extra buffer tank. In this case we assume that the latter alternative is the best and introduce a new tank after the mixing tank (dashed in Fig. 3). We see from Fig. 4 that the gain must be reduced with 10 at the bandwidth $(f=10)$, and obtain a required residence time of the buffer tank of $20 \mathrm{~s}$ from (24) $(n=1)$. The modified disturbance transfer function gain, $\left|G_{\mathrm{d}}\right|$, is shown with a solid line in Fig. 4. The slope is -1 or smaller below the bandwidth, so that we need not consider the problem discussed in a subsection under Controllability. $|L(j \omega)|$ is plotted (dash-dotted) to illustrate this $\left(\left|G_{\mathrm{d}}\right|<|L|\right)$. $\left|S G_{\mathrm{d}}\right|$ is below 1 for all frequencies (dashed). Fig. 5 shows the response of a unit step in concentration of stream $A$ with (solid) and without (dashed) the extra buffer tank. We see that it is kept below 0.1 with the extra buffer tank present.

If the slope of $\left|G_{\mathrm{d}}\right|$ is steeper than the slope of $|L|, \tau_{\mathrm{h}}$ is too optimistic. We will however analyze one case. We assume $\left|G_{\mathrm{d}_{0}}\right|$ has slope -1 so that $\left|G_{\mathrm{d}}\right|$ has slope -2 above the frequency $1 / \tau_{\mathrm{h}}$, where $\tau_{\mathrm{h}}$ is the buffer tank residence time. Further, we assume that $|L|$ has slope -1 near the bandwidth and that it increases to -2 due to an integrator in the controller below $\omega=1 / \tau_{\mathrm{I}}$, where $\tau_{\mathrm{I}}$ is the integral time. A robust choice of $\tau_{\mathrm{I}}$ is $8 \theta_{\text {eff }}$ (Skogestad, 1999). Using geometry it is easy to 
show that in this case $\tau_{\mathrm{h}}=8 f \theta_{\text {eff. }}$. Compared to (24) for one tank we see that the residence time for this case is increased by a factor of 8 .

Example 2. Consider the process from Example 1, modified so that the measurement delay is $0.1 \mathrm{~s}$, the volume of the first tank is $5 \mathrm{~m}^{3}$ and the variation requirements for the outlet concentration is 0.01 . The concentration in the first tank is controlled with a robust PI controller (Skogestad, 1999). In this case the slope of $\left|G_{\mathrm{d}}(j \omega)\right|$ is -2 around the bandwidth, and (24) leads to a residence time of $0.39 \mathrm{~s}$, which is insufficient. In Fig. 6 a residence time of $\tau_{\mathrm{h}}=8 f \theta_{\text {eff }}=$ $3.2 \mathrm{~s}$ is applied. The method uses asymptotes, and we see that $\left|G_{\mathrm{d}}(j \omega)\right|$ is just touching the asymptote of $|L(j \omega)| .|L(j \omega)|$ itself is a distance above $\left|G_{\mathrm{d}}\right|$ so the result here is slightly conservative. By optimization one finds a minimum residence time of $2.4 \mathrm{~s}$ required to fulfil (21) for this controller tuning.

\section{Flow variations}

By exploiting the volume of the buffer tank, flow

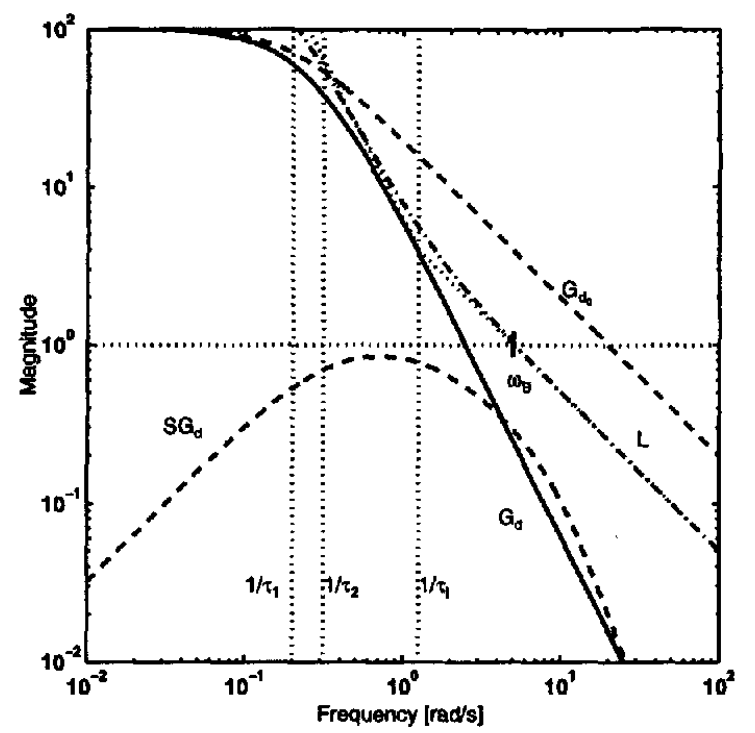

Fig. 6. With a residence time of $\tau_{\mathrm{h}}=8 f \mathrm{olr}_{\mathrm{er}}$ in the second tank, $|L(j(\omega))|>\left|G_{\mathrm{d}}(j \omega)\right|$ for all frequencies and disturbances are rejected.

Table 1

Procedure for buffer tank design applied to first- and second-order filtering

\begin{tabular}{lll}
\hline Step & 1 st order & 2nd order \\
\hline 1. Desired $h(s)$ & $1 /\left(\tau_{1} s+1\right)$ & $1 /\left(\tau_{2} s+1\right)^{2}$ \\
2. $k(s)$ from $(11)$ & $1 / \tau_{1}$ & $\frac{1}{2 \tau_{2}} \frac{1}{\frac{\tau_{2}}{2} s+1}$ \\
3. $V(0) / q_{\text {in }},(0)$ from $(27) \tau_{1}$ & & $2 \tau_{2}$ \\
4. $V_{\text {tot }}$ & & $2 \tau_{2} \Delta q_{\max }$ \\
\hline
\end{tabular}

variations in the outflow may be dampened using a slow level control. The outflow will then be dependent on the chosen controller. Denote the tank volume $V$ $\left(\mathrm{m}^{3}\right)$ and the inlet and outlet flow-rates $q_{\text {in }}$ and $q$, respectively. The transfer function for the buffer tank is then given by (10). Compared with the quality disturbance case, we have more freedom in selecting $h$, since we can select the controller $k(s)$. But the level will vary, so the size of the tank must be chosen so that the level remains between its limits. The volume variation is given by $V(s) / q_{\text {in }}(s)=1 /(s+k(s))$, and combination with (11) yields:

$V(s) / q_{\text {in }}(s)=(1-h(s)) / s$

which is used to find the required tank volume. The tank size design consists of the following steps:

1. Select $h(s)$ such that if has the desired shape, that is such that (16) is satisfied.

2. Find the corresponding controller from (11) (is it realizable?)

3. Find the largest effect of $q_{\text {in }}$ in $V$ from (27) (usually at steady state, $s=0$ ).

4. Obtain the required total volume from the expected range of $q_{\text {in }}$ (denoted $\Delta q_{\text {in }}$ ).

In Table 1 we have applied the method for first and second order filtering.

\subsection{First-order filtering}

With $h(s)=1 / \tau_{1} s+1$ the required controller is a P-controller with gain $K_{c}=1 / \tau_{1}$. From (27), $V(s)=\left(\tau_{1} /\right.$ $\left.\tau_{1} s+1\right) q_{\text {in }}(s)$. The maximum value of this transfer function occurs at low frequencies $(s=0)$, and the required volume of the tank is $V_{\text {tot }}=\tau_{1} \Delta q_{\max }$. Adding a slow integral action to the controller will not affect these results considerably. Such an integral action will reset the volume to its nominal value. This is not always desired, however. If for example $q_{\text {in }}$ is at its maximum, we may want the volume to stay at a large value to anticipate a possible large reduction in $q_{\text {in }}$.

\subsection{Second-order filtering}

With $h(s)=1 /\left(\tau_{2} s+1\right)^{2}$ we get from (11) that the required controller is a lag

$$
k(s)=\frac{1}{2 \tau_{2}} \frac{1}{\frac{\tau_{2}}{2} s+1}
$$

and from (27) the response of the volume deviation is

$$
V(s)=2 \tau_{2} \frac{\left(\tau_{2} / 2\right) s+1}{\left(\tau_{2} s+1\right)^{2}} q_{\text {in }}(s)
$$

This has its largest value equal to $2 \tau_{2}$ at low frequencies $(s=0)$, and the required volume is $2 \tau_{2} \Delta q_{\max }$. 


\section{References}

Harriott, P. (1964). Process control. New York: McGraw-Hill. Lieberman, N. P. (1983). Process design for reliable operations. Houston: Gulf Publishing Company.

McMillan, G. K. (1984). pH control. NC, USA: Instrument Society of America.

Sandler, H. J., \& Luckiewicz, E. T. (1987). Practical process engineering. New York: McGraw-Hill.

Shinskey, F. G. (1973). pH and pIon control in process and waste streams. New York: Wiley.

Sigales, B. (1975). How to design reflux drums. Chemical Engineering, $82(5), 157-160$.

Skogestad, S. (1999). Lecture notes for the course Process control (Tillegg til fag 52041 Prosessregulering, in Norwegian).
Skogestad, S., \& Postlethwaite, I. (1996). Multivariable feedback control. Chichester, New York: Wiley.

Ulrich, G. D. (1984). A Guide to chemical engineering process design and economics. New York: Wiley.

Walas, S. M. (1987). Rules of thumb, selecting and designing equipment. Chemical Engineering, 94(4), 75-81.

Walsh, S. (1993). Integrated design of chemical waste water treatment systems. Ph.D thesis. Imperial College, UK.

Watkins, R. N. (1967). Sizinffig separators and accumulators. Hydrocarbon Processing, 46(11), 253-256.

Wells, G. L. (1986). The art of chemical process design. Amsterdam: Elsevier.

Zheng, A., \& Mahajanam, R. V. (1998). A quantitative controllability index. Internal report; Similar presented at 1997 American Institute of Chemical Engineering Annual Meeting, LA. 